\title{
Phenomenon of self-consciousness and its development during infancy and childhood
}

\author{
Maria Kondratiuk ${ }^{1, *}$ \\ ${ }^{1}$ Leningrad State University, 196605, Petersburg Highway, 10, Saint Petersburg, Russia
}

\begin{abstract}
The purpose of this work is determining the features of selfconsciousness development in infancy and childhood. The main research methods are analysis and synthesis. The role of self-awareness and personality is evaluated. The importance of self-awareness in ontogenetic development is shown. The work shows that the development of selfconsciousness begins with infancy, when the first prerequisites for its development arise, and the first formed elements of self-consciousness are manifested in preschool childhood. The dynamics of the development of self-consciousness during infancy and childhood. is shown. Considers the key aspects of its development during infancy, early childhood and preschool age. The special role of the environment and communication for the formation of self-awareness is studied. The development of elements of self-awareness in preschool age is characterized. The essential internal and external conditions for development of self-consciousness within these age periods were analyzed.
\end{abstract}

\section{Introduction}

Over the decades, the problem of self-consciousness has been remaining relevant within the academic community. Self- consciousness as a scientific problem has a long history. Since Antiquity, consciousness and self- consciousness have been at the center of philosophical discussions, and to this day the question of self- consciousness occupies the minds of many scientists. Initially, ideas about self-consciousness developed in the mainstream of philosophy, since ancient times this concept was an open topic of discussion and was associated with consciousness, thinking, perception, but modern philosophy has refused to identify these concepts. Currently, philosophical views on the problem of selfconsciousness are turned towards the existing possibility of reflection of behavior; and selfconsciousness is considered not just as a combination of certain «self-knowledge», but also experiences about the content of subjective reality. And actually the genesis, components, factors that influence the formation of self-consciousness in ontogenesis and its place among other psychological phenomena, is studied by psychology. Today, selfconsciousness is a puzzle to solve for a set of both humanities and social sciences, since it is a comprehensive phenomenon and there is no general consensus on the phenomenology of consciousness. The question "What is self-consciousness?" may be answered depending on certain constructs, in terms of which this concept is considered. Poor comprehension of

\footnotetext{
${ }^{*}$ Corresponding author: maria kondratiuk@bk.ru
} 
self-consciousness is caused by intricacy of its structure, its multilevel nature and lack in systematic description of its components and their functioning, as well as by semantic and contextual ambiguousness of the term [1].

The issue of self-consciousness formation is important in modern conditions, as the consciousness of the self contributes to the formation of a unique inner world and worldview, self-development and self-determination, building a harmonious relationship with the world around us. There is social success, the importance of a person as a representative of society, capable of independent choice, successful resolution of difficulties, having an active position depends on the level of self-consciousness development [2]. Self- consciousness is one of the most hidden phenomena of the human psyche. It generally reflects the way of existence of the individual and its subjective reality, as well as an indicator of the relationship between the individual and the world, gives a person an idea of their place in the environment.

\section{Literature analysis}

In psychology, self-consciousness acts as a purely personal formation that substantially determines the psychological and social appearance of a person, his position and mode of existence in the world. Despite the great interest in the topic of self-consciousness, there is no single understanding of this phenomenon, features of its development and a generally accepted definition. The insufficient elaboration of the problem of self-consciousness is caused by the ambiguity of its structure, multi-levelness, the absence of systematic descriptions of its components and their functioning, the semantic and meaningful fragmentation of this concept. It is worth noting that the study of the dynamics of the development of self-consciousness is still of great interest, both theoretically and in practical terms, since it allows you to get closer to understanding the mechanisms of personality formation in ontogenesis [3].

«The Principles of Psychology» by William James is deemed to be a starting point of studies on self-consciousness in psychology. He considered self-consciousness as a term differing from personal identity, supposed that it gives a start to all psychological processes and serves as a tool to process all received experience, while perceiving oneself and one's personality was supposed to be permanent [4]. Thereafter William James's theory was elaborated, criticized and reconsidered, new opinions on self-consciousness phenomenon arose. Looking at self-consciousness from the point of view of various psychological directions, it can be concluded that in behaviorism, self-consciousness is a behavioral category that is found in actions; psychoanalysis connects the appearance of the self with the separation (in the literal and figurative sense) of the child from the mother, consciousness and self-consciousness are opposed to the unconscious, acting as a certain sphere of mental life, in cognitive approaches, self-consciousness is associated with the presence of a cognitive scheme by which a person reorganizes information about himself in special concepts and images; in interactionism, self-consciousness is the result of communication; and from the point of view of humanistic and existential approaches, selfconsciousness acts as a personality of a construct developed by a healthy, developed one, scientists of these areas consider the essence of the human self through the prism of selfactualization and creativity. Today via analysis and synthesis of various perspectives it can be concluded that self-consciousness has the following important characteristics:

- self-consciousness is an integral entity and an essential part of consciousness, arising and freely developing while being inextricably bounded up with consciousness.

- self-consciousness is both a process and the result of this process [5].

- self-consciousness is not a congenital attribute, it forms gradually due to the involvement of a person into a society and one's interaction with it. And this is the point 
where one faces the paradox of self-consciousness: attitude to oneself forms through his or her attitude to others, a person learns to perceive oneself through another person. According to $\mathrm{Ph}$. Rochat, a human is obsessed with the idea of publicness, concerned of what others may think of him or her as of a person or a member of a group, which he or she identifies him- or herself with (family, group, nation, culture). Consciousness is mediated by others and results from «cogitamus, ergo sum» [6];

- self-consciousness is an open multicomponent entity, thereat, all its structural components having complex configuration and special functions comprise a united act of self-consciousness. According to V. S. Mukhina, the following aspects stand out of structure of self-consciousness: identification with one's name and body; claim on acceptance; gender identity; psychological age of a person; social space.

- processes of self-consciousness involve the whole personality, not only the combination of separate individual mental processes.

\section{Results}

Self- consciousness is a complex mental process that manifests itself, first of all in a person's perception of numerous «images» of himself in various situations of activity and communication. These «images» initially arise on the basis of a given person's awareness of his assessments by other people, then on the basis of correlation of assessments of others and his own. In other words, they are always the result of the social interaction of the individual and his environment. Primary images of oneself, rich in sensory content, are integrated into a single holistic entity - into the conception, and then into the concept of the self as a subject different from others. In the movement from the individual, situational images of oneself to the identification of the essential and the unique, the process of selfknowledge is carried out. Self-consciousness ensures the experience of feeling unity and specificity of one's self-concept, integrates personality and supports one's internal consistency. Due to self-consciousness, one can perceive and estimate oneself as an individual. Self-consciousness helps a person to form self-consistency in his or her assessments and actions, to know oneself and serves as the source of continuous selfidentity through the time. Digestion, interpretation and integration of experience occur through self-consciousness and its structural components, furthermore it is selfconsciousness what determines one's expectations and serves as the source of selfdevelopment and self-improvement. Self- consciousness is one of the most significant internal conditions for the continuity of the personality genesis, which allows you to establish a balance between the internal processes occurring in the personality, the behavior chosen by it and the external environmental influences $[7,8,9]$.

Self-consciousness does not appear in one moment like a point on an ontogenesis line. It develops gradually, step-by-step. It is worth mentioning that the presence of selfconsciousness is attributed to rational self-esteem arising at the age of 7 , however thorough consideration of earlier periods proves the presence of some elements of self-consciousness at a much younger age.

Coming to life, a baby does not aware of oneself, of who he or she is, cannot localize one's feelings, since this work requires self-consciousness. Further elaborating and improving during one's life, self-consciousness will finally enable finding an answer to the enternal "Who am I?" question. During infancy, development of self-consciousness is attributed to the continuously increasing activities, formation of spatial relations, development of motor and senses.

During first months of one's life a baby does not understand the difference between oneself and the environment, does not distinguish oneself as a separate being, but day by day the environment helps a baby in his or her development and supports the beginning of 
his or her self-consciousness. Early age period is fairly important since it involves the arising of self-concept and imbedding of emotion-and-value component of selfconsciousness, which further makes a strike way to synthetic self-esteems and integral selfconcept and self-image. In this regard, ability to distinguish and perceive emotional state of adults surrounding a baby is vitally important during the infancy period, as long as it gives a boost to the development of empathy, which is an essential quality for a harmonious personality with a high level of self-consciousness. This requires an adequate feedback from people around; communication with adults showing wide spectrum of emotions allows a child to get acquainted with and form an image of those emotions and the way how to express them. Such emotional communication bends baby's cognitive activity to him- or herself as well as to surrounding objects. In the period of infancy cognitive and affective self-images are poorly formed, yet they exist in the shape of gaining emotionally positive experience of one's own subjectivity while interacting with others. Perception of one's own body is related to the midpoint of infancy; within the same period scrutiny of other people's behavior pattern starts. Feeling oneself being within boundaries of one's own body is the initial form of further self-consciousness that forms on the basis of tactual sense modality, kinesthetic and equilibrium sense [10]. Child's kinesthetic activity promotes the development of not only elements of self-consciousness during infancy, but his or her mental development in general.

The main achievement of infancy period is gaining the ability to walk that brings a child to the next level, which often goes together with shifting to the stage of early childhood - a period when substantive activity starts to prevail, speech development begins and framework of communicative behavior is established - all these aspects give a boost to selfconsciousness development. When 18 months old, a child is already able to pass the mirror test, which indicates the initial discrimination of him- or herself from the rest of the world. The reactions of children of different ages to the mirror are well studied and are a behavioral indicator of the development of self-awareness. Based on these reactions, $\mathrm{Ph}$. Rocha identifies 6 chronological levels of development of self-consciousness from birth to early childhood:

Level 0 - confusion: lack of self-consciousness; at this stage, mirror reflection is perceived as a continuation of the environment.

Level 1 - differentiation: implicit self-consciousness, differentiation; there is a distinction between the environment and the reflected, its own reflection in motion and the movement of the rest are realized.

Level 2 - situation: the appearance of a contemplative position on the mirror image self-examination.

Level 3 - identification: recognition of oneself in the mirror, the emergence of the ability to correlate with one's own body of mirror reflection; this level indicates the beginning of the formation of the image of the self.

Level 4 - permanence: recognizing onerself not only here and now in the mirror, but also in photographs or videos in other conditions.

Level 5 - self-consciousness or "meta" self-awareness: the ability to present oneself through the eyes of other people, the appearance of secondary emotions of shame and pride, the manifestation of these emotions in front of a mirror; children begin to understand they can be in the mind of others - how they are seen from a public standpoint [6].

Mirror self-recognition mediates the appearance of self-consciousness. Synthesis of two selves - the subjective I, who is in front of the mirror, and the objective Me, the reflected one, is a pivotal point in mental development [11]. At the age of two, continuously growing willfulness and independence allows children to get to know themselves and the environment, a child already has the understanding that he or she is the exact initiator of his or her own activity, gets the feeling of independence, child's activities transform into some 
sort of self-sufficiency. Early childhood is the key period for mindset forming, this particular period is associated with embedding the course for self-esteem that will affect the self-concept during the whole life of a person. Self-consciousness of a child is especially exposed to parents' judgments that become a significant aspect of self-image and selfesteem formation, since communication with parents takes almost all time of a child. Development of self-consciousness is based on discrimination of oneself from others, and one of the ways to do it is naming, to which a child forms a certain attitude. Furthermore, naming is the basis for one particular component of self-consciousness: concept of one's own name. All indicative addresses to a child by his or her name are the markers that can become a basis for the behavior pattern, yet not only naming but whatsoever estimation of an adult pushes a child towards a certain self-concept or self-image.

As the result, passing through infancy and early childhood, a child obtains the components of self-consciousness, which becomes possible due to gradual gathering of prerequisites for its development. Figure 1 presents self-consciousness development dynamic. The self-consciousness can be proclaimed when a child starts to discriminate the process and the result of his or her activities from those of others' and is able to oppose himself to others. The emergence of expression "I can do it myself" indicates the ability to distinguish between oneself and one's aims and actions which promotes the development of true independence, goal-setting, ambitions and relative self- sufficiency. The expression of will and understanding one's own initiative, which means understanding one's feelings and elementary impulses, concept of one's own body and one's visual image - all these attributes indicate the emergence of initial self-consciousness, when a child perceives oneself as a separate person, active subject and a human being with one's unique Self. It is worth noting that self-consciousness is formed during infancy and childhood only due to the environment surrounding a child, and due to his or her interaction with this environment. During further age stages, the development of self-consciousness depends on internal activity and orientation, one's own processing of information about oneself, not just its receiving. Thus, the environment and people around determine self-consciousness of a child during infancy and childhood.

Preschool childhood is a period of intense mental development. This ontogenetic stage involves the transformation of all spheres: psychophysiological functions are being improved, personality development is proceeding, need for communication with peers and demand for socially significant activity appear, creative activity and ability to selfregulation are developing, the attitude towards the world is being rebuilt, a child is actively mastering his own body, higher psychic functions are developing, child's activities are becoming more complex, the readiness for school is being formed [9].

Passing to the stage of preschool childhood, the child has certain knowledge about himself, his properties, qualities, capabilities, which accumulate due to constant activity and communication. In the period of preschool age, more and more independence and independence is gained. The child's opportunities are expanding, relations are becoming more complicated and more saturated. The main activity at this stage is a game that allows you to try on different roles and simulate all kinds of situations. At the age of 4-7 years, an outline of the worldview arises - an integral schematic picture of the environment. A preschooler seeks to explain and organize information about the world in his mind. Activities are guided by a system of motives, and the ability to look from the perspective of another is the basis of many skills.

Self-attitude and self-image are formed due to the integration of personal experience and feedback, which is the information obtained during communication. It is during preschool age, when such a component of self-consciousness as self-esteem starts taking its initial shape and self-image keeps forming. The accuracy of the child's self-image corresponds to the adequacy of adult estimates, so underestimated estimates will distort the 
child's self-image, leading to distrust or refusal of activity, overestimated estimates can both distort perceptions of oneself, contributing to the emergence of unrealistic expectations, and contribute to mobilization and stimulate optimism, confidence, support [12]. Communication with adults and their assessments are the main resource of child's ideas about not only him- or herself, but also the world and people. The interaction between a child and an adult contributes to the enrichment of individual experience, shows a way to solve certain problems, promotes the processing, systematization and generalization of gained knowledge, and also serves as a way to compare oneself to an ideal - an adult. At this stage a child gets some initial ideas of his or her future, forms an image of what he or she may become when growing up.

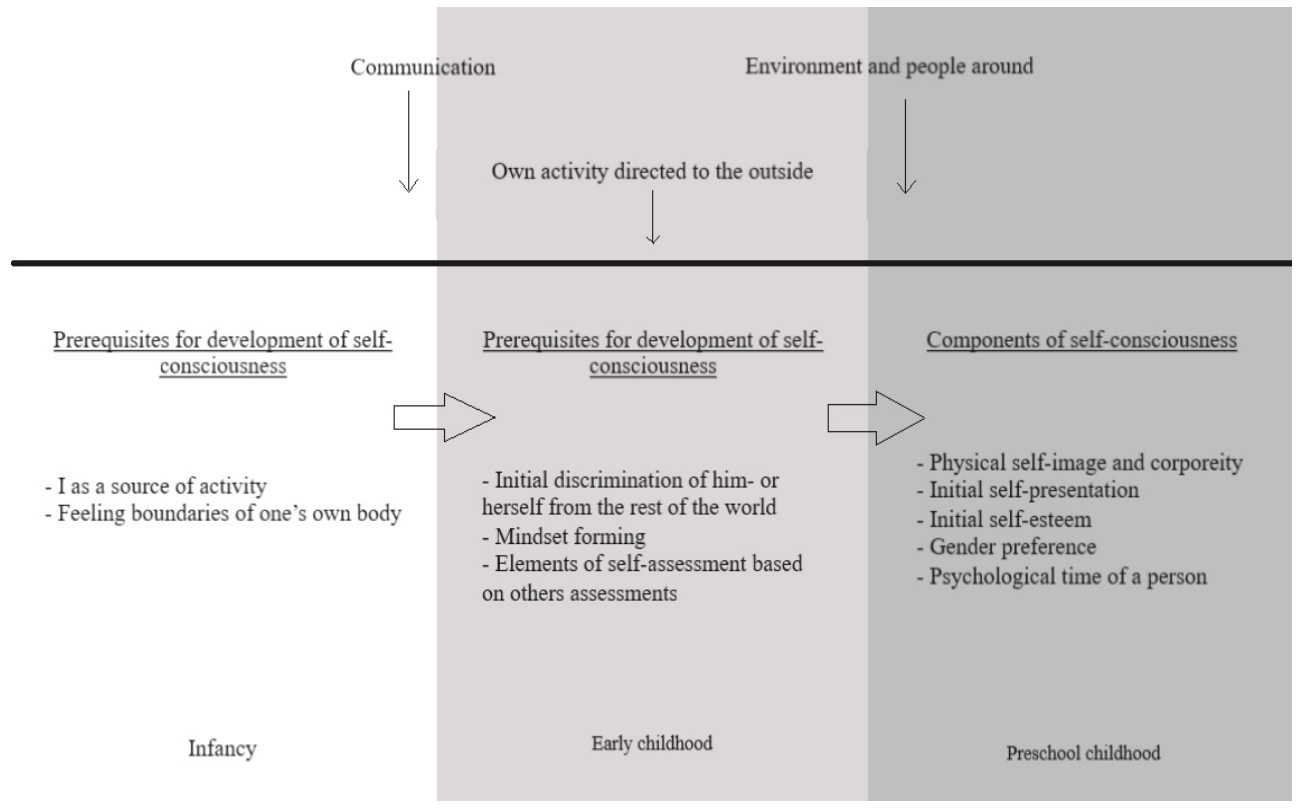

Fig. 1. Self-consciousness development dynamic.

Such self-knowledge mechanisms as identification and reflection through communication with peers are emerging. Communication in a team of peers serves as a means of exchanging assessments through which the child receives feedback from peers and sees himself through their eyes. Of great importance in this period are business qualities, success in a collective activity, moral qualities - all these characteristics are considered by preschoolers themselves, but peer analysis is more practical and objective when self-esteem is more situational and emotional. If development during preschool childhood undergoes properly, self-concept actively expands, self-image improves, selfconsciousness goes beyond the framework of object characteristics, it becomes possible to estimate oneself, to understand both oneself and others.

The main moral self-regulation factor is self- consciousness, and the main motive for observing generally accepted norms is the desire to maintain a positive self-image; it is on the basis of this desire that by the middle of preschool age, children are able to regulate their behavior on the basis of social norms in a situation of moral choice, give a moral assessment of actions, understand other people's experiences. The emergence of moral judgments indicates the differentiation of self-consciousness into the I-real and I-ideal, the opposition of the image of good and bad, but the learned norm does not always correspond to behavior, and, therefore, has not yet been appropriated by the child as a personal one. 
By the preschool age, a child has already got a clearly formed idea of and attitude to one's own name, a child also has got certain ideas of one's own body and attitude to it: everything of this creates the first fundamental link in the structure of self-consciousness. A person's name serves as a social sign, individualizes a person and, as long as development proceeds, acquires twisty integrative connections with various mental entities, entailing the formation of self-attitude, certain claims and much more things. Representation and attitude to one's own body form a body image, physical self-image and corporeity; they take a great part in self-acceptance and in the structure of self-consciousness in general.

The next formed structural element of self-consciousness is the claim on acceptance, which is formed due to the emotionally-evaluative relationship between an adult and a child. During first seven years of ontogenetic development, there is an active assimilation of behavior patterns, interests and values regarding one's gender through imitation, which will subsequently play a significant role in the development of self-consciousness. Understanding of the separation of bad and good by a child pushes him to actions that are approved. The claim for recognition acts as a presentation to others of their rights to respect by society, and is the need of a person as a social being. The deprivation of this need in preschool age can contribute to the appearance of lies and envy, the manifestation of aggression, insecurity, passivity, conformity, and contribute to the growth of internal tension and frustration [13].

By the start of preschool childhood, a child has a clear idea of his own belonging to a certain gender and is able to recognize others' genders, yet from the perspective of external signs, such as clothing, makeup, hairstyle. Moreover, preschoolers are aware of the fact that certain requirements are imposed on a person depending on one's gender. It is worth noting that a preschooler has peculiarities regarding his or her gender identification: the assessment of one's own masculinity-femininity is complicated by blurred sex-role preferences. Preschoolers definitely divide themselves into boys and girls and on the basis of this they build their behavior, prefer certain classes and certain colors. In games, preschoolers have the opportunity to simulate the behavior characteristic of both men and women, but this does not mean "throwing" or ignorance of their own gender. During the first seven years of ontogenetic development, the assimilation of behavioral forms, interests, and values of one's sex through imitation is actively taking place, which will subsequently play a significant role in self-awareness of oneself.

Such a link in the self-consciousness structure as psychological time of a person does already exist and actively develops within the period of preschool childhood. Psychological time of a person implies the awareness of one's change over time - during the past, present and future. The emergence of psychological time is manifested by child's thoughts of what he or she will be like when grown up, or what he or she was like before; psychological time of a person becomes a measuring scale indicating any change and development. Indeed, a preschool child has the uncritical and naive scale, but still it serves as an incentive that inspires one for improving and achieving goals. In addition to the personal component, psychological time involves awareness of one's family and national history. It is the preschool period, when children become persistently familiarized with culture and history, which helps them form ethnic self-consciousness.

Factors affecting the development of self-consciousness in the period of preschool childhood - these factors can be divided into both internal and external. Internal factors include:

- self-awareness as a subject of activity and community;

- internal activity;

- a set of motives, including the motive of self-correspondence.

But external factors are of particular importance in preschool age: 
1. Social space: place of residence, features of culture and ethnos, style and content of communication in the context of culture, generally accepted values and norms - all this contributes to the development of personality and self-awareness in particular;

2. Features of family education and relationships - the preschooler sees himself through the eyes of significant adults; provided that the estimates and expectations of adults do not match the age and individual characteristics, the child's self-image is distorted. A democratic parenting style, respect for the child, his interests, a positive assessment of abilities, frequent encouragement, but not gifts, are a contribution to an adequate selfesteem and a positive image of oneself;

3. The game, as the leading activity at this age, contributes to the development of selfregulation, in the game there is a development and satisfaction of the need for selfaffirmation. Games expand the preschooler's self-image, because it is in the course of interpersonal interaction with classmates without adult participation that the ability to establish and build contacts, fulfill roles, and defend one's opinion is manifested.

4. The influence of the media, specifically - various cartoons, broadcasts, and also printed children's products — translated ready-made images, standards, values and behaviors in a form accessible to the child from the outside are assimilated and transformed into personal ones.

5. Preschool educational institution - the majority of preschoolers attend kindergartens, which contribute to the development of self-awareness through certain means:

- spatial-subject equipment, which stimulates all types of activity of the child. A wellorganized object-spatial environment provides an opportunity for active interaction and joint activities, encourages conscious choice, and teaches to put forward and implement their own initiatives and solutions. In the conditions of a preschool educational institution, a child independently strives for activities that develop his mental and creative abilities, imagination, communication skills, and shape the personality as a whole;

- the personality of the kindergarten teacher, who becomes a particularly significant figure for the pupils: the teacher contributes to the development of the child's self-esteem, characterizing him; Paying attention to changes in the child, his achievements, successes, development dynamics, the educator contributes to the formation of the psychological time of the individual. The teacher of a preschool educational institution becomes an example for a child, and since preschool children have developed imitation, a personal example of a teacher is put aside in the minds of children, processed and becomes their personal. The kindergarten teacher takes part in the formation of morality, lays the foundation for morality and ethics, and forms value orientations by means of evaluation, explanation, and making demands. The teacher also contributes to the development of the child's selfesteem, characterizing him as a whole, his actions and results. In addition, the kindergarten teacher has a huge impact on the children's team; the behavior of children, their emotional state, microclimate in the group, the perception of each other and themselves personally depend on the personality characteristics of the teacher;

- peer team - the child takes a certain position in the team and begins to understand the attitude of other children to himself; he also critically evaluates peer behavior from his peers as equal to himself, which makes it possible to adequately evaluate himself, highlight the «position of another», and change the egocentric picture of the world $[14,15]$.

Within the preschool age, prerequisites for development of the fifth element of selfconsciousness are just initially embedded. When getting knowledge of social norms and acquiring morality principles, a child is not yet aware of one's rights and duties, which are detached from one's personality and have obscure image, but their acceptance becomes the basis for understanding the structure of society and one's place in a social space. 


\section{Discussion}

Preschoolers are receptive and sensitive to existential experiences, feelings of the boundaries of the self, position among peers, and the experience of their own uniqueness. The importance and sensitivity of the self at this age makes preschoolers vulnerable to outside influence; a friendly, reliable and non-aggressive environment is a resource for the development of preschoolers. Features of an environment where a child lives, standards, social principles and a community greatly influence the formation of self-consciousness. During infancy and childhood, social space determines the formation of self-consciousness and a person as an individual, affecting both his or her social and personal component. This process undergoes as long as a child accepts material and non-material culture, values, social standards and attitudes, as well as communicates with adults who direct him or her and embed information about oneself, and is engaged with peers who serve as objects to compare oneself with and reflect oneself. Sensitivity peculiar to a child during one's infancy and childhood makes one highly vulnerable in terms of the formation of one's selfesteem, self-attitude and self-consciousness, therefore a kind and reliable environment is a required source for one's development.

\section{References}

1. D. Zahavi, T. Grünbaum, J. Parnas, The structure and development of selfconsciousness: interdisciplinary perspectives, 162 (2004)

2. J. L. Bermúdez, The Paradox of Self-consciousness (A Bradford Book, 2000)

3. P. Carruthers, Phenomenal consciousness. A naturalistic theory (Cambridge University Press, 2000)

4. J. William, The Principles of Psychology, 1, 708 (Cosimo, Inc., 2007)

5. A. Morin, Laterality: Asymmetries of Brain, Behaviour, and Cognition, 16, 367-383 (2011) 10.1080/13576501003702648

6. P. Rochat, Others in Mind: social origins of self-consciousness (Cambridge University Press, 2009)

7. J. V. Canfield, Becoming human: the development of language, self, and selfconsciousness (Palgrave Macmillan, New York, 2007)

8. R. J. Bogdan, Our Own Minds: Sociocultural Grounds for Self-Consciousness (A Bradford Book, 2010)

9. K. Adolph, S. Berger, Handbook of child psychology (NY, Wiley, 2006)

10. C. A. Brownell, S. Zerwas, G. B. Ramani, Child Development, 78(5), 1426-1440 (2007) 10.1111/j.1467-8624.2007.01075

11. G.G. Gallup, J.L. Anderson, D.P. Shillito, "The mirror test," in The Cognitive Animal: Empirical and Theoretical Perspectives on Animal Cognition (University of Chicago Press, 325-333)

12. R. Banerjee, Merrill-Palmer Quarterly, 378-404 (2002)

13. G. L. Brown, S. C. Mangelsdorf, et al., Wayne State Univ. Press, 55(2), 184-216 (2009)

14. E. S. Buhs, Journal of School Psychology, 43(5), 407-424 (2005) 10.1016/j.jsp.2005.09.001

15. S. Settea, D. Baldwinb, et al., Early Childhood Research Quarterly 47, 229-238 (2019) 10.1016/j.ecresq.2018.12.012 